\title{
Reproductive function in male rats with chronic nephrosis
}

\author{
G. Ortiz ${ }^{1}$, F. Vilchis ${ }^{2}$, M. Cárdenas ${ }^{2}$,C. Cruz ${ }^{3}$, J. Pedraza-Chaverrí ${ }^{4}$ and M. Menjívar ${ }^{4 *}$ \\ ${ }^{1}$ Department of Reproductive Biology, Hospital Juárez de México, Mexico City, Mexico; ${ }^{2}$ Department of Reproductive Biology and \\ ${ }^{3}$ Department of Nephrology, Instituto Nacional de la Nutrición Salvador Zubirán, Mexico City, Mexico; and ${ }^{4}$ Department of Biology, \\ Faculty of Chemistry, Universidad Nacional Autónoma de México, Mexico City, Mexico
}

\begin{abstract}
Endocrine dysfunction has been associated with renal diseases. The present study was conducted to explore reproductive function in male rats with chronic nephrosis. Experimental chronic nephrosis was induced by the administration of 7.5, 5.0 and 5.0 $\mathrm{mg}$ per $100 \mathrm{~g}$ body weight of puromycin aminonucleoside on days 0,21 and 35 , respectively. Reproductive function was evaluated on the basis of hormonal concentrations, mass of accessory sex organs and fertility during an 84 day period. Circulating LH, FSH, testosterone and oestradiol concentrations were measured by specific radioimmunoassays, while fertility was estimated by the rate of pregnancy induction. Samples were collected on days $7,14,28,56$ and 84 . The results showed an important endocrine dysfunction characterized by low concentrations of LH and FSH during the first month, after which concentrations were similar to control values or even increased on days 56 and 84 . Testosterone and oestradiol decreased significantly at all time points evaluated. The mass of the testes did not alter. However, the mass of the prostate and seminal vesicle decreased only during the first 2 weeks, and became essentially normal thereafter. The reproductive capacity of nephrotic males was eliminated on day 7 , whereas on day $14,16 \%$ of the group was able to mate successfully and subsequently most animals recovered their normal reproductive function. This study demonstrates for the first time that rats with experimental chronic nephrosis develop an important endocrine dysfunction, characterized mainly by persistent reduction in testosterone concentrations, which impairs reproductive capacity only transiently.
\end{abstract}

\section{Introduction}

Nephrotic syndrome in rats and humans is characterized by alterations in the metabolism of plasma proteins (Glassock et al., 1991). In addition, there is evidence that male nephrotic animals also develop a marked endocrine dysfunction (Glass et al., 1984, 1985; Elias et al., 1992). In previous reports this condition has been described in male and female nephrotic rats during the acute phase of the illness (Menjivar et al., 1995, 1997). These findings showed that nephrotic animals developed hypogonadism-hypogonadotropic, characterized mainly by low LH and testosterone concentrations, but also by undetectable oestradiol concentrations, follicular atresia, undetectable oestrous cycle, a decrease in the mass of male accessory sex organs, and a decrease in reproductive capacity.

The administration of puromycin aminonucleoside (PAN), which induces oxidant-mediated podocyte injury

*Correspondence.

Received 16 November 1998.
(Diamond et al., 1986), has been used to develop both acute and chronic nephrosis. The chronic stage of nephrosis is a lesion of focal segmental glomerulosclerosis (FSGS), which in humans is characterized by a steroid-resistant and progressive deterioration of renal function (Glassock and Brenner, 1994). The pathogenesis of the FSGS is unknown, and the resistance to glucocorticoid therapy necessitates the use of immunosuppressive agents as well as cytotoxic compounds; these can have adverse effects on bone marrow, the gonads and the bladder. In this context, it is clear that further studies are required to clarify the pathogenesis and determine more adequate treatment for the nephrotic patient.

After the acute stage of nephrotic syndrome, a remission period, in which most of the endocrine alterations are reverted, is presented (Glass et al., 1985). Thus, it was hypothesized that persistent proteinuria, which is the main characteristic of nephrotic syndrome, leads to a chronic endocrine dysfunction, which results in chronic reproductive changes. The present study was designed to evaluate the hormonal status of male rats with induced chronic nephrosis, and its effect on reproductive capacity. 


\section{Materials and Methods}

\section{Reagents}

Puromycin aminonucleoside (PAN) was purchased from Sigma Chemical Co. (St Louis, MO). Reagents for radioimmunoassay of LH and FSH were kindly provided by the National Hormone and Pituitary Program (NIH) (Baltimore, MD). Radioimmunoassay kits for testosterone and oestradiol were purchased from Diagnostic Products Corporation (Los Angeles, CA). All other chemicals were of reagent grade and available commercially.

\section{Animals}

Adult male Wistar rats weighing $280 \pm 12 \mathrm{~g}$ in control groups and $282 \pm 10 \mathrm{~g}$ in nephrotic groups were maintained under a $14 \mathrm{~h}$ light: $10 \mathrm{~h}$ dark photoperiod. The animals were fed with Lab Diet brand animal food, containing not less than $23 \%$ crude protein from PMI Feeds (St Louis, MO). Water and food pellets were available ad libitum. For the fertility evaluation, groups of female animals showing three or more consistent 4 day oestrous cycles were included in the study. Body weight was also measured at all time points.

\section{Experimental design}

Chronic nephrosis was induced by the administration of 3 s.c. doses of PAN at 7.5, 5.0 and $5.0 \mathrm{mg}$ per $100 \mathrm{~g}$ body weight on days 0,21 and 35, respectively. The control group received a sham injection of saline. Rats were observed for an 84 day period. Blood samples and urine samples over $24 \mathrm{~h}$ were collected on days $7,14,28,56$ and $84(n=7$ animals at each time point). Samples were stored at $-20^{\circ} \mathrm{C}$ until assayed. The prostate, seminal vesicles and testes were excised, cleaned and weighed at each time point.

\section{Fertility evaluation}

The fertility of nephrotic and control animals was evaluated at each established time point in the additional groups, by keeping rats individually in cages for mating with one normal female for a 4 day period as described by Menjívar et al. (1997). When mating and fertilization occurred, the number of pregnant rats as well as the weight, sex and litter size were recorded.

\section{Sperm count}

The number of spermatozoa present in the cauda epididymis was determined in nephrotic and control groups on days 7 and 14. The left and right caudae epididymides from each rat were minced with scissors and gently homogenized in $1 \mathrm{ml}$ saline solution $(0.9 \%)$. The sperm count per cauda epididymis was determined as described by Amann (1981).

\section{Hormonal assay}

The serum LH and FSH were measured by specific radioimmunoassay using the double antibody technique with reagents and protocol supplied by NIH. The intra- and interassay coefficients of variations were $5.1 \%$ and $6.5 \%$ for $\mathrm{LH}$ and $4 \%$ and $7.9 \%$ for $\mathrm{FSH}$, respectively. The results are expressed in $\mathrm{ng} \mathrm{ml}^{-1}$ of the international reference standards NIH LH-RP-3 and FSH-RP-2. Steroid hormones, testosterone and oestradiol concentrations were also measured by radioimmunoassay. The intra- and interassay coefficients of variation were $4.4 \%$ and $8.2 \%$ for testosterone and $6.1 \%$ and $7.4 \%$ for oestradiol, respectively.

\section{Analytical determinations}

Total protein in serum and urine as well as cholesterol and creatinine in serum were measured by spectrophotometric methods as described by Pedraza-Chaverrí et al. (1993) and Menjívar et al. (1995).

\section{Statistical analysis}

The unpaired Student's $t$ test or the Mann-Whitney U tests were used to assess significance. $P<0.05$ was considered significant.

\section{Results}

The results for the $24 \mathrm{~h}$ proteinuria, cholesterol, creatinine and serum protein in control and nephrotic animals during the investigation are summarized (Table 1). The urinary excretion of protein was significantly higher in nephrotic animals than in controls at all time points. Similarly, serum cholesterol was significantly higher in nephrotic animals than in controls. Although the values of total serum protein were lower in the nephrotic animals than in controls during the first month of the study, there were no differences between groups at any of the subsequent time points. Serum creatinine remained unchanged in both groups.

The body weight of nephrotic and control rats was recorded to evaluate the impact of chronic nephrotic syndrome on growth rate. The body weight of nephrotic animals was significantly lower than that of non-treated rats at all time points (Table 2). However, neither testis mass (Table 2) nor sperm count was affected by nephrotic syndrome (day 7: nephrotic $=146 \times 10^{6}$ versus control $=$ $144 \times 10^{6}$ spermatozoa per cauda epididymis; day 14 : nephrotic $=190 \times 10^{6}$ versus control $=186 \times 10^{6}$ spermatozoa per cauda epididymis).

The main serum hormone concentrations evaluated for 84 days are presented (Fig. 1). The data indicate that $\mathrm{LH}$ decreased significantly only on day 7 , and subsequently $\mathrm{LH}$ concentrations were similar to, or greater than, control values. Similarly, FSH concentrations, which decreased on days 7,14 and 28 , were not different from control values at the subsequent time points. Gonadal steroid hormones 
Table 1. Biochemical determinations in serum and urine from control (C) and nephrotic $(N)$ rats

\begin{tabular}{|c|c|c|c|c|c|c|}
\hline Day & Group & $\begin{array}{l}\text { Urine protein } \\
\text { (mg per } 24 \text { h) }\end{array}$ & $\begin{array}{l}\text { Serum protein } \\
\qquad\left(\mathrm{g} \mathrm{dl}^{-1}\right)\end{array}$ & $\begin{array}{l}\text { Serum cholesterol } \\
\qquad\left(\mathrm{mg} \mathrm{dl}^{-1}\right)\end{array}$ & $\begin{array}{l}\text { Serum creatinine } \\
\left(\mathrm{mg} \mathrm{dl}^{-1}\right)\end{array}$ & $n$ \\
\hline \multirow[t]{2}{*}{7} & C & $28 \pm 3$ & $7.6 \pm 0.4$ & $61 \pm 2$ & $0.4 \pm 0.00$ & 6 \\
\hline & $\mathrm{N}$ & $533 \pm 53^{*}$ & $4.9 \pm 0.1^{*}$ & $313 \pm 14^{*}$ & $0.6 \pm 0.06$ & 7 \\
\hline \multirow[t]{2}{*}{14} & C & $28 \pm 3$ & $7.5 \pm 0.1$ & $62 \pm 3$ & $0.4 \pm 0.00$ & 6 \\
\hline & $\mathrm{N}$ & $437 \pm 31^{*}$ & $6.9 \pm 0.2^{*}$ & $263 \pm 11^{*}$ & $0.4 \pm 0.04$ & 7 \\
\hline \multirow[t]{2}{*}{28} & C & $25 \pm 2$ & $8.1 \pm 0.2$ & $57 \pm 3$ & $0.4 \pm 0.01$ & 7 \\
\hline & $N$ & $499 \pm 44^{*}$ & $5.6 \pm 0.2^{*}$ & $219 \pm 29^{*}$ & $0.4 \pm 0.04$ & 6 \\
\hline \multirow[t]{2}{*}{56} & C & $30 \pm 9$ & $6.9 \pm 0.1$ & $64 \pm 2$ & $0.5 \pm 0.00$ & 7 \\
\hline & $\mathrm{N}$ & $449 \pm 65^{*}$ & $6.7 \pm 0.2$ & $435 \pm 79^{*}$ & $0.6 \pm 0.08$ & 7 \\
\hline \multirow[t]{2}{*}{84} & C & $19 \pm 3$ & $9.5 \pm 0.2$ & $75 \pm 4$ & $0.7 \pm 0.01$ & 6 \\
\hline & $\mathrm{N}$ & $653 \pm 132^{*}$ & $8.8 \pm 0.3$ & $165 \pm 26^{*}$ & $0.7 \pm 0.05$ & 7 \\
\hline
\end{tabular}

Chronic nephrosis was induced by the administration of puromycin aminonucleoside (PAN) on days 0,21 and 35 . Results are expressed as mean \pm SEM. ${ }^{*} P<0.05$ versus controi group.

Table 2. Body weight and testicular mass of control (C) and nephrotic (N) rats

\begin{tabular}{ccccc}
\hline Day & Group & Body weight $(\mathrm{g})$ & Testis mass $(\mathrm{g})$ & $n$ \\
\hline \multirow{2}{*}{7} & $\mathrm{C}$ & $326 \pm 5$ & $1.84 \pm 0.16$ & 6 \\
& $\mathrm{~N}$ & $299 \pm 5^{*}$ & $1.82 \pm 0.20$ & 7 \\
& $\mathrm{C}$ & $299 \pm 8$ & $1.70 \pm 0.12$ & 6 \\
28 & $\mathrm{~N}$ & $242 \pm 8^{*}$ & $1.63 \pm 0.11$ & 7 \\
& $\mathrm{C}$ & $370 \pm 9$ & $1.80 \pm 0.27$ & 7 \\
56 & $\mathrm{~N}$ & $300 \pm 5^{*}$ & $1.85 \pm 0.13$ & 7 \\
& $\mathrm{C}$ & $410 \pm 9$ & $1.80 \pm 0.13$ & 7 \\
& $\mathrm{~N}$ & $289 \pm 15^{*}$ & $1.60 \pm 0.28$ & 6 \\
& $\mathrm{C}$ & $475 \pm 12$ & $1.86 \pm 0.12$ & 7 \\
\hline
\end{tabular}

Chronic nephrosis was induced by the administration of puromycin aminonucleoside (PAN) on days 0,21 and 35 . Results are expressed as mean \pm SFM. ${ }^{*} P<0.05$ versus control group.
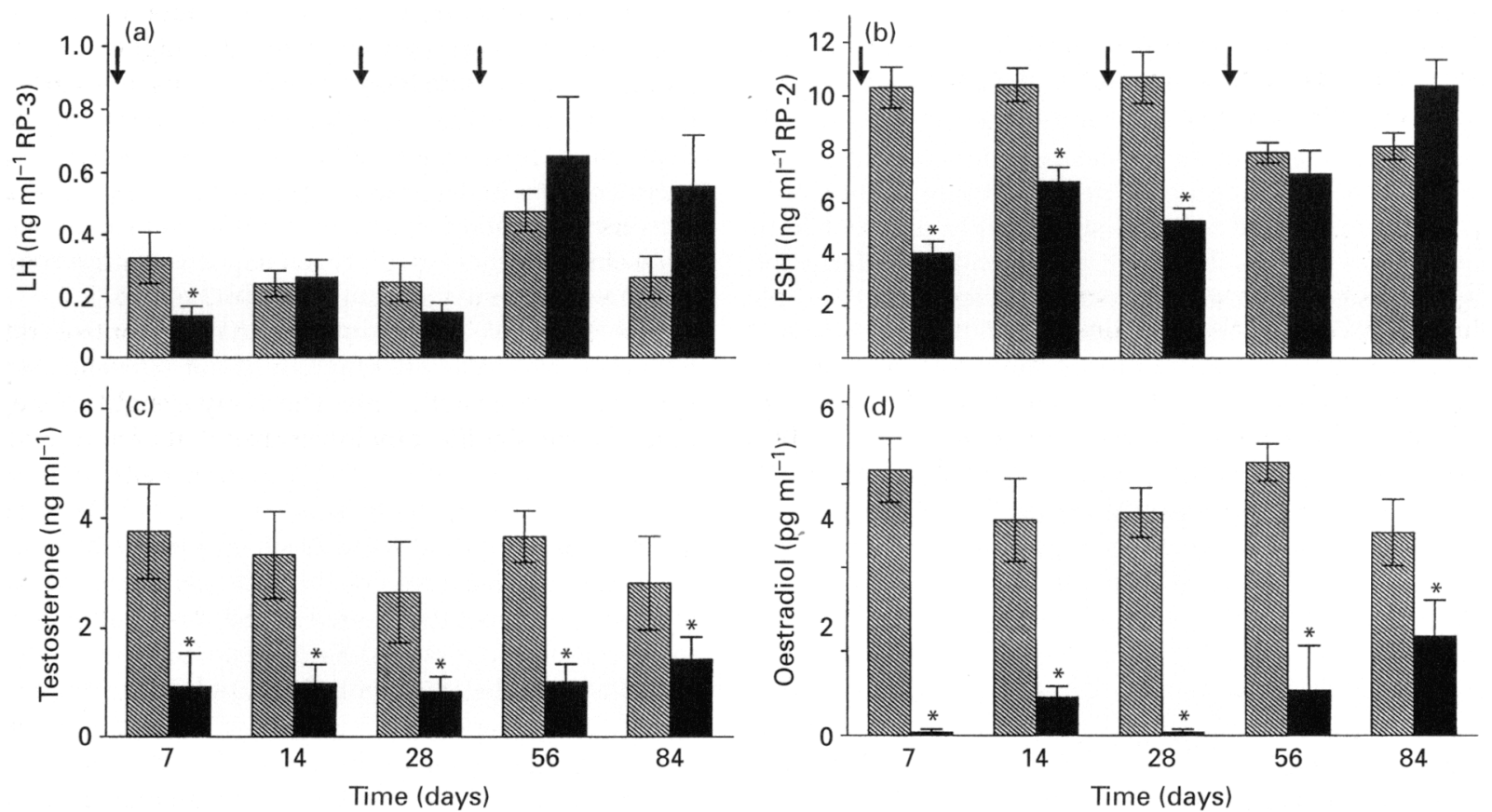

Fig. 1. Serum concentrations of (a) LH, (b) FSH, (c) testosterone and (d) oestradiol from control ( $\mathbb{N}$ ) and nephrotic ( $\mathbf{\square}$ ) rats. Arrows show the times when puromycin aminonucleoside (PAN) was administered (days 0,21 and 35 ). Values are expressed as mean \pm SEM. ${ }^{*} P<0.05$ versus control group. 

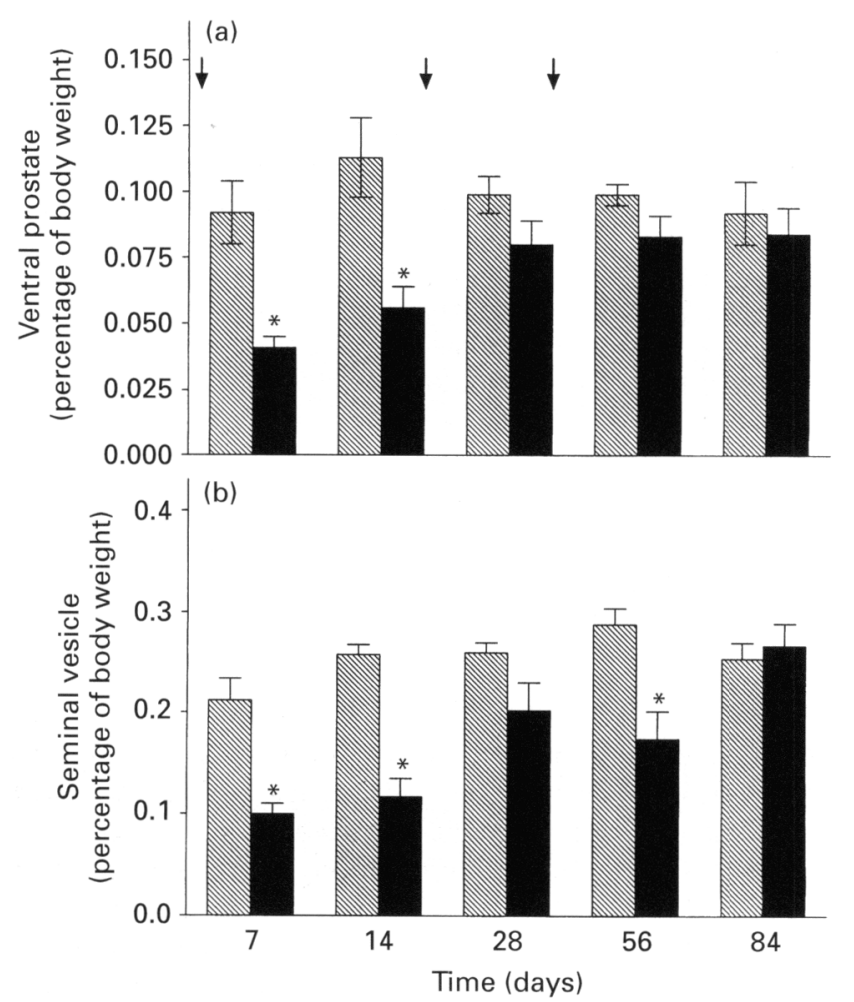

Fig. 2. Mass of (a) ventral prostate and (b) seminal vesicle expressed as a percentage of body weight in control ( and nephrotic (ם) rats. Arrows show the time when puromycin aminonucleoside (PAN) was administered (days 0,21 and 35). Values are expressed as mean \pm SEM. ${ }^{*} P<0.05$ versus control group.

decreased significantly in nephrotic groups at all time points.

The masses of the seminal vesicle and ventral prostate expressed as a percentage of body weight are shown (Fig. 2). As expected, although the mass of the sex accessory organs decreased in response to the low testosterone concentrations, the mass of the ventral prostate decreased significantly only on days 7 and 14, and was not significantly different from control values at the subsequent time points. Similarly, the mass of the seminal vesicle decreased on days 7,14 and 56 , and was in the range of the controls at the other time points.

The reproductive capacity of male nephrotic and control animals is shown (Table 3 ). The percentage fertility as well as the litter characteristics showed that none $(0 / 7)$ of the nephrotic males were fertile on day 7 , and only $16 \%(1 / 7)$ were able to mate successfully on day 14. After day 14 , nephrotic rats recovered their reproductive capacity to within the range observed in control animals, which was between 71 and $85 \%$.

\section{Discussion}

This study demonstrates that male rats with induced chronic nephrosis developed a clear endocrine dysfunction. This condition was characterized by a persistent reduction in testosterone concentrations and an inappropriate gonadotrophin response during the study. The hormonal disorders had only a transient effect upon the mass of sex accessory organs and on fertility.

In the biochemical evaluation, proteinuria, which is the main characteristic of nephrotic syndrome, was observed throughout the 84 days of the study. However, the concentration of total serum protein returned to control values after day 28 despite the proteinuria. Sierra et al. (1997) suggested that this effect might be an adaptive response to compensate for urinary protein excretion.

Menjívar et al. (1997) reported that endocrine disorders in the acute stage of nephrotic syndrome appear to be reversible. In contrast, in the present study of chronic nephrosis, the reduction in sex steroid hormones, which was the major endocrine alteration, was constant. At all time points studied, testosterone and oestradiol concentrations were significantly lower in the nephrotic rats, thus indicating an alteration in gonadal steroid production. However, since there was suppression of the hypothalamic-pituitary-gonadal axis and decreased concentrations of both gonadotrophins and testosterone, a failure in the feedback control of the hypothalamic-pituitary-gonadal axis is postulated, including a failure in GnRH secretion, impairment of gonadotrophin synthesis or release, and impairment of the gonadal response to gonadotrophins through their specific testicular receptors. Most studies concerned with the association between renal diseases and disturbances in gonadal function have demonstrated that hypogonadism is principally due to aberrant hypothalamic regulation of pituitary $\mathrm{LH}$ secretion (Handelsman et al., 1985; Veldhuis et al., 1993a,b; Schaefer et al., 1994). In this context, it may be hypothesized that hypothalamic-pituitary alterations might be the initial disturbance or a key factor which leads to the reduction in testosterone concentrations during chronic nephrosis.

In the present study, the testis mass was similar between the nephrotic and control groups at all time points. These results are in agreement with those of Glass et al. (1985), who showed that testicular histology is not affected by the acute stage of nephrotic syndrome. In addition, no differences were found in sperm counts in the cauda epididymis on days 7 and 14 between nephrotic and control animals. This can be explained by the low threshold of androgen required to preserve normal spermatogenesis in the adult rat (Buhl et al., 1982).

Menjivar et al. (1997) reported that male rats with acute nephrotic syndrome presented hypoandrogenism associated with the atrophy of accessory sex glands. In the present study on induced chronic nephrosis, the mass of the sex accessory organs, the ventral prostate and the seminal vesicle, decreased initially by about $50-55 \%$ (days 7 and 14), but subsequently tended to reach control values. Thus, in contrast to expected results, the androgen-dependent tissues were not permanently affected by the chronic hypogonadism in the nephrotic animals. Since male accessory organs are essentially under the control of androgen through their specific receptors (Liao et al., 1975), and androgenic deprivation in the rat prostate upregulates the concentration of transcripts for the androgen receptor (Kumar et al., 1997), it was hypothesized that an androgen receptor-mediated mechanism may explain this unexpected finding. However, the involvement of non-androgenic factors in the control of 
Table 3. Reproductive function in control $(\mathrm{C})$ and nephrotic $(\mathrm{N})$ rats

\begin{tabular}{ccccccc}
\hline Day & Group & Fertility rate & Fertility $(\%)$ & $\begin{array}{c}\text { Number of offspring } \\
\text { per litter }\end{array}$ & Sex ratio F:M $(\%)$ & Weight $(\mathrm{g})^{\mathrm{ab}}$ \\
\hline 7 & $\mathrm{C}$ & $7 / 7$ & 100 & $11 \pm 4$ & $54: 46$ & $35 \pm 6$ \\
\multirow{3}{*}{14} & $\mathrm{~N}$ & $0 / 7$ & 0 & 0 & 0 & 0 \\
& $\mathrm{C}$ & $5 / 7$ & 71 & $12 \pm 3$ & $47: 53$ & $49 \pm 6$ \\
28 & $\mathrm{~N}$ & $1 / 7$ & 16 & $10 \pm 0$ & $40: 60$ & $55 \pm 3$ \\
& $\mathrm{C}$ & $5 / 7$ & 71 & $12 \pm 2$ & $43: 57$ & $51 \pm 4$ \\
56 & $\mathrm{~N}$ & $6 / 7$ & 85 & $10 \pm 3$ & $49: 51$ & $50 \pm 3$ \\
& $\mathrm{C}$ & $6 / 7$ & 85 & $12 \pm 3$ & $51: 49$ & $51 \pm 3$ \\
& $\mathrm{~N}$ & $6 / 7$ & 85 & $10 \pm 3$ & $47: 53$ & $50 \pm 3$ \\
& $\mathrm{C}$ & $6 / 7$ & 85 & $10 \pm 3$ & $50: 50$ & $49 \pm 5$ \\
\hline
\end{tabular}

Chronic nephrosis was induced by the administration of puromycin aminonucleoside (PAN) on days 0,21 and 35.

Values are mean \pm SD.

'Body weight at day 21 after birth.

the accessory sex glands, such as epidermal growth factor, transforming growth factors $\alpha$ and $\beta$, and fibroblast growth factors, which are positive and negative regulators of prostate growth and function (Culig et al., 1996), cannot be ruled out. Furthermore, Brewster et al. (1997) reported that oestrogen activity was involved in the maintenance of the seminal vesicle. The involvement of oestradiol cannot explain the unexpected finding of the present study, since the oestradiol concentration was affected to a greater extent than testosterone. In the nephrotic group, there was a clear relationship between the masses of the ventral prostate and the seminal vesicle. However, the difference observed in the seminal vesicle mass on day 56 could be explained by the observation that these tissues are modulated by testosterone in an organ-specific manner (Fahim et al., 1993; Huang et al., 1997). Collectively, the findings of the present study show that rats with chronic nephrosis are able to overcome steroid deprivation to maintain the normal mass of accessory sex glands as well as reproductive function.

Although the relationship between multisystemic diseases and fertility is well established (Handelsman, 1985; Steger and Rabe, 1997), the adverse effect of chronic nephrosis on reproductive function is not well recognized and has not been studied. In the present study, the effect of chronic nephrosis on fertility and progeny characteristics was investigated. Reproduction was affected only in nephrotic rats on days 7 and 14 , when the fertility rate decreased to $0 \%$ $(0 / 7)$ and $16 \%(1 / 7)$, respectively. At the subsequent time points, all nephrotic rats were able to mate successfully. These data indicate that the hypogonadic-hypogonadotrophic condition and the small mass of the accessory sex organs reduced or completely eliminated reproductive capacity on days 7 and 14. However, normal reproductive capacity was possible even with greatly reduced hormonal support on days 28-84. Menjívar et al. $(1995,1997)$ demonstrated that acute nephrosis is characterized by a harmful effect on the reproductive capacity of both male and female rats. In addition, da Costa e Silva et al. (1984) reported a prevalence of infertility in uremic rats. Thus, the reduction in reproductive capacity on days 7 and 14 followed by complete recovery in the nephrotic animals was unexpected, mainly because there was a high correlation of reproductive capacity with the mass of accessory sex organs, rather than with testosterone concentrations. Furthermore, testosterone remained at a low concentration throughout the study, and it is known that without gonadotrophins, a higher than normal concentration of serum testosterone is required to maintain normal fertility (Buhl et al., 1982). This finding may be explained by the adaptive response involving an androgen receptor-mediated mechanism proposed earlier (Liao et al., 1975; Kumar et al., 1997). Alternatively, the normal sperm count of nephrotic animals observed in the present study on days 7 and 14 may be responsible for these findings. Further studies are required to define the specific mechanisms involved in chronic nephrosis.

The recovery of fertility during chronic nephrosis, together with the low or normal LH concentrations and the low oestradiol concentrations observed in this study, as well as in the acute stage of nephrosis (Menjívar et al., 1995, 1997), enabled nephrotic syndrome to be distinguished from chronic renal failure. It is well known that chronic renal failure is characterized by high $\mathrm{LH}$ concentrations, normal oestradiol concentrations and low fertility (Handelsman et al., 1985; Handelsman and Dong, 1993; Yamamoto et al., 1996).

With regards to offspring, no adverse effects were observed on all litter characteristics, nor were any significant nephrosis-related differences found in the duration of gestation, sex ratio, mean number or weight of pups per litter. These findings are similar to those reported in the acute stage of nephrotic syndrome (Menjivar et al., 1997) and in rats suffering from chronic renal failure (Yogev et al., 1993). Thus, it is concluded that in the experimental models of these renal diseases, there is no apparent effect of the renal and endocrine alterations on the progeny.

Growth failure has been also related to kidney diseases. Growth retardation is considered a central feature of chronic renal failure in children (Trachtman et al., 1993). Furthermore, abnormalities in the systemic and renal insulin-like growth factor system are known to occur in nephrotic syndrome, as in chronic or acute renal failure (Feld and Hirschberg, 1996; Hirshberg and Adler, 1998). In agreement with these reports, the results of the present study showed that chronic 
nephrosis has an adverse effect on weight gain; growth retardation, on the basis of body weight measurements, was apparent at all time points.

In conclusion, the results presented here establish that an important endocrine dysfunction is associated with chronic nephrosis. This endocrine dysfunction has two components: the first, which is probably irreversible, is characterized by low testosterone concentrations and a failure in the gonadotrophin response; and the second, which is reversible, is marked by a transitory impairment of the sex accessory glands and fertility.

This research project was supported by Grants from PAPIT (No. IN201596) National University of Mexico and CONACYT (No. 25126-M), Mexico City, México. The authors wish to thank the National Hormone and Pituitary Program (Bethesda, MD) for providing rat $\mathrm{LH}$ and FSH biological materials for the performance of assays.

\section{References}

Amann RP (1981) A critical review of methods for evaluation of spermatogenesis from seminal characteristics Journal of Andrology 237-58

Brewster ME, Anderson WR and Pop E (1997) Effects of sustained estradiol release in the intact male rat: correlation of estradiol serum levels with actions on body weight, serum testosterone, and peripheral androgendependent tissues Physiology and Behaviour 61 225-229

Buhl AE, Cornette JC, Kirton KT and Yuan YD (1982) Hypophysectomized male rats treated with polydimethylsiloxane capsules containing testosterone: effects on spermatogenesis, fertility, and reproductive tract concentrations of androgens Biology of Reproduction 27 183-187

Culig Z, Hobisch A, Cronauer MV, Radmayr C, Hittmair A, Zhang J, Thurnher M, Bartsch G and Klocker H (1996) Regulation of prostatic growth and function by peptide grow th factors Prostate 28 392-405

da Costa e Silva A, Ribeiro RCJ, Albuquerque RH, Beraldo PSS, Neves FAR, Martinelli JG and Campos GP (1984) Effect of experimentally induced renal failure upon the fertility in uremic rats Nephron 36 252-256

Diamond JR, Bonventre JV and Karnovsky MJ (1986) A role for oxygen free radical in aminonucleoside nephrosis Kidney International 29 478-483

Elias AN, Carreon G, Vaziri ND, Pandian MR and Oveisi F (1992) The pituitary-gonadal axis in experimental nephrotic syndrome in male rats Journal of Laboratory and Clinical Medicine 120 949-954

Fahim MS, Wang M, Sutcu MF and Fahim Z (1993) Zinc arginine, a 5 alphareductase inhibitor, reduces rat ventral prostate weight and DNA without affecting testicular function Andrologia 25 369-375

Feld S and Hirschberg R (1996) Growth hormone, the insulin-like growth factor system, and the kidney Endocrine Reviews 17 423-480

Glass AR, Vigersky RA, Rajatanavin R, Pardridge W, Smallridge RC and Wartofsky $L$ (1984) Low serum thyroxine and high serum triiodothyroxine in nephrotic rats: etiology and implications for bioavailability of proteinbound hormone Endocrinology 114 1745-1753

Glass AR, Beach J and Vigersky RA (1985) Hypogonadotropic hypogonadism in nephrotic rats: increased sensitivity to negative feedback effects of testosterone Metabolism 34 574-579

Glassock RJ and Brenner BM (1994) The major glomerulopathies. In Harrison's Principles of Internal Medicine pp 1295-1306 Eds KJ Isselbache, E Braunwald, J Wilson, JB Martin, AS Fauci and DL Kasper. McGraw-Hill, New York
Glassock RJ, Adler SG, Ward HJ and Cohen AH (1991) Primary glomerular diseases. In The Kidney pp 1182-1253 Eds BM Brenner and FC Rector. WB Saunders, Philadelphia

Handelsman DJ (1985) Hypothalamic-pituitary gonadal dysfunction in renal failure, dialysis and renal transplantation Endocrine Reviews 6 151-182

Handelsman DJ and Dong Q (1993) Hypothalamo-pituitary-gonadal axis in chronic renal failure. In Endocrinology and Metabolism Clinics of North America. Neuroendocrinology II pp 145-161 Ed JD Velduis. WB Saunders, Philadelphia

Handeisman DJ, Spaliviero JA and Turtle JR (1985) Hypothalamic-pituitary function in experimental uremic hypogonadism Endocrinology 117 1984-1995

Hirshberg R and Adler S (1998) Insuline-like growth factor system and kidney: physiology, and therapeutic implications American Journal of Kidney Diseases $31901-919$

Huang HF, Li MT, Von Hagen S, Zhang YF and Irwing RJ (1997) Androgen modulation of the messenger ribonucleic acid of retinoic acid receptors in the prostate, seminal vesicle, and kidney in the rat Endocrinology 138 553-559

Kumar VL, Majumder PK and Kumar RV (1997) Androgen deprivation causes up-regulation of androgen receptor transcript in the rat prostate Molecular Cell Biochenistry 171 133-138

Liao S, Tymoczko JL, Castañeda E and Liang T (1975) Androgen receptors and androgen-dependent initiation of protein synthesis in the prostate Vitamins and Hormones 33 297-317

Menjívar M, Vilchis F, Cardenas M, Cruz C, Merchant H, Pérez-Palacios G and Pedraza-Chaverrí J (1995) Pituitary-ovarian dysfunction in rats with nephrotic syndrome European Journal of Endocrinology 132 502-506

Menjivar M, Vilchis F, Cardenas M, Cruz M, Perez-Palacios G and PedrazaChaverrí J (1997) Transient alteration of the reproductive function in nephrotic rats Life Sciences 61 1159-1167

Pedraza-Chaverrí J, Calderón P, Cruz C and Peña JC (1993) Electrophoresis analysis of serum and urinary proteins in rats with aminonucleosideinduced nephrotic syndrome Renal Failure 15 149-155

Schaefer F, Daschner M, Veldhuis JD, Oh J, Qadri F and Scharer K (1994) In vivo alterations in the gonadotropin-releasing hormone pulse generator and the secretion and clearance of luteinizing hormone in the uremic castrate rat Neuroendocrinology 59 285-296

Sierra RI, Specker BL, Jimenez F, Cruz C and Pedraza-Chaverrí J (1997) Biochemical bone markers, bone mineral content, and bone mineral density in rats with experimental nephrotic syndrome Renal Failure 19 402-424

Steger RW and Rabe MB (1997) The effect of diabetes mellitus on endocrine and reproductive function Proceedings of the Society for Experimental Biology and Medicine 214 1-11

Trachtman H, Futterweit S, Schwob N, Maesaka J and Valderrama E (1993) Recombinant human growth hormone exacerbates chronic puromycin aminonucleoside nephropathy in rats Kidney International 44 1281-1288

Veldhuis JD, Iranmanesh A, Evans WS, Lizarralde G, Thorner MO and Vance ML (1993a) Amplitude suppression of the pulsatile mode of immunoradiometric luteinizing hormone release in fasting-induced hypoandrogenemia in normal men Journal of Clinical Endocrinology and Metabolism 76 587-593

Veldhuis JD, Wilkowski MJ, Zwart AD, Urban RJ, Lizarralde G, Iranmanesh $A$ and Bolton WK (1993b) Evidence for attenuation of hypothalamic gonadotropin-releasing hormone (GnRH) impulse strength with preservation of $\mathrm{GnRH}$ pulse frequency in men with chronic renal failure fournal of Clinical Endocrinology and Metabolism 76 648-654

Yamamoto Y, Sofikitis N and Miyagawa I (1996) Effects of chronic renal failure on hypothalamo-pituitary-testicular axis function and fertility in rats International Journal of Urology 3 484-490

Yogev L, Serban I, Benjamin G, Yavets H, Homonnai Z, Cabili S, Iaina A and Paz G (1993) Experimental uremia in male rats: effect on the reproductive tract and fertility Renal Failure 15 131-134 